\title{
NEW RELATIONS AMONG EULER SUMS OF EVEN WEIGHT
}

\|\|

\author{
WEN-CHIN LIAW
}

Abstract. In this paper, we shall consider different kinds of Euler sums which are related to Ramanujan's constant $G(1)$. We develop new relations among these Euler sums and classical Euler sums of even weight. In particular, from these relations we give explicit evaluations of

$$
G_{1,2 n+1}=\sum_{k=0}^{\infty} \frac{1}{(2 k+1)^{2 n+1}} \sum_{j=1}^{k} \frac{1}{j} .
$$

\section{Introduction}

For a pair of positive integers $p$ and $q$ with $q>1$, the classical Euler sum is defined as

$$
S_{p, q}=\sum_{k=1}^{\infty} \frac{1}{k^{q}} \sum_{j=1}^{k} \frac{1}{j^{p}} .
$$

See for example 1, 2, 3, 5] for the details. The following well-known formula for $S_{1, n}$ is due to Euler (cf. 1, p. 253]).

Theorem A. For each positive integer $n$ with $n \geq 2$, we have

$$
S_{1, n}=\frac{n+2}{2} \zeta(n+1)-\frac{1}{2} \sum_{j=2}^{n-1} \zeta(j) \zeta(n+1-j) .
$$

In an attempt to investigate relations among Euler sums of the same even weight, Chen and Eie [4] defined, for positive integers $p$ and $q$ with $q \geq 2$, some related new sums as

$$
\begin{aligned}
G_{p, q} & =\sum_{k=0}^{\infty} \frac{1}{(2 k+1)^{q}} \sum_{j=1}^{k} \frac{1}{j^{p}} \\
H_{p, q} & =\sum_{k=0}^{\infty} \frac{1}{(2 k+1)^{q}} \sum_{j=0}^{k} \frac{1}{(2 j+1)^{p}}
\end{aligned}
$$

Received December 21, 2006.

2000 Mathematics Subject Classification. Primary 11M06; Secondary 11B68.

Key words and phrases. Euler sums, even weight. 
and for positive integers $r$,

$$
E_{p, q}^{(r)}=\sum_{k=1}^{\infty} \frac{1}{k^{q}} \sum_{j=1}^{k r} \frac{1}{j^{p}} .
$$

These generalized Euler sums come naturally from $S_{p, q}$. For example,

$$
\begin{aligned}
S_{p, q} & =\sum_{k=1}^{\infty} \frac{1}{k^{q}} \sum_{j=1}^{k} \frac{1}{j^{p}} \\
& =\sum_{k=1}^{\infty} \frac{1}{(2 k)^{q}} \sum_{j=1}^{2 k} \frac{1}{j^{p}}+\sum_{k=0}^{\infty} \frac{1}{(2 k+1)^{q}} \sum_{j=1}^{2 k+1} \frac{1}{j^{p}} \\
& =2^{-q} E_{p, q}^{(2)}+H_{p, q}+2^{-p} G_{p, q} .
\end{aligned}
$$

In his famous notebooks 1 , p. 252], Ramanujan claimed that

$$
G(1):=\sum_{k=1}^{\infty} \frac{1}{8 k^{3}} \sum_{j=1}^{k} \frac{1}{2 j-1}=\frac{\pi}{4} \sum_{k=0}^{\infty} \frac{(-1)^{k}}{(4 k+1)^{3}}-\frac{\pi}{3 \sqrt{3}} \sum_{k=0}^{\infty} \frac{1}{(2 k+1)^{3}} .
$$

The above formula is incorrect as can be easily seen numerically. In our notation we have

$$
8 G(1)=E_{1,3}^{(2)}-\frac{1}{2} S_{1,3}=E_{1,3}^{(2)}-\frac{5}{8} \zeta(4)=G_{2,2}+\frac{15}{16} \zeta(4) .
$$

There is no closed-form evaluation known for $E_{1,2 n+1}^{(2)}$, nor for $G_{p, q}$ if $p+q$ is even. However, we are able to evaluate $G_{1,2 n+1}$ and $E_{1,2 n+1}^{(2)}+2^{2 n+1} H_{1,2 n+1}$. Here are our main theorems in this paper.

Theorem 1. For each positive integer n, we have

$$
\begin{aligned}
G_{1,2 n+1}= & \left(2-2^{-(2 n+1)}\right)\left\{S_{1,2 n+1}-\zeta(2+2 n)\right\} \\
& -2 \lambda(2 n+1) \log 2+\sum_{\ell=2}^{2 n} 2^{1-\ell} \zeta(\ell) \lambda(2 n+2-\ell),
\end{aligned}
$$

where

$$
\lambda(s)=\sum_{k=0}^{\infty} \frac{1}{(2 k+1)^{s}}=\left(1-2^{-s}\right) \zeta(s) .
$$

Theorem 2. For each positive integer n, we have

$$
\begin{aligned}
& E_{1,2 n+1}^{(2)}+2^{2 n+1} H_{1,2 n+1} \\
& =\frac{1}{2} S_{1,2 n+1}+\left(2^{2 n+1}-\frac{1}{2}\right) \zeta(2 n+2)+2^{2 n+1} \lambda(2 n+1) \log 2 \\
& \quad-\sum_{\ell=2}^{2 n} 2^{2 n+1-\ell} \zeta(\ell) \lambda(2 n+2-\ell) .
\end{aligned}
$$


As a byproduct of our results, we also prove the following relations among Euler sums of the same even weight.

Theorem 3. For each positive integer $n$,

$$
\begin{aligned}
\sum_{\ell=2}^{2 n} 2^{\ell-1} S_{\ell, 2 n+2-\ell}= & \left(2^{2 n}-1\right) S_{1,2 n+1}-2^{2 n} \zeta(2 n+2) \\
& +\sum_{\ell=2}^{2 n} 2^{2 n+1-\ell} \zeta(\ell) \lambda(2 n+2-\ell) .
\end{aligned}
$$

Two more general theorems will be given in Section 5 .

\section{The evaluation of $G_{1,2 n+1}$}

As shown in (1.6), we already have a formula relating $G_{1,2 n+1}$ to $E_{1,2 n+1}^{(2)}+2^{2 n+1}$ $H_{1,2 n+1}$. We need one more relation in order to evaluate $G_{1,2 n+1}$. First we obtain an evaluation of $G_{1,2 n+1}$ in terms of $S_{\ell, 2 n+2-\ell}$ for $\ell=2,3, \ldots, 2 n$.

Proposition 1. For each positive integer n, we have

$$
\begin{aligned}
G_{1,2 n+1}= & \left(1+2^{-(2 n+1)}\right) S_{1,2 n+1}-\left(1-2^{-(2 n+1)}\right) \zeta(2 n+2) \\
& -2 \lambda(2 n+1) \log 2+2^{-2 n} \sum_{\ell=2}^{2 n} 2^{\ell-1} S_{\ell, 2 n+2-\ell} .
\end{aligned}
$$

Proof. We begin with $E_{1,2 n+1}^{(2)}$ defined by

$$
E_{1,2 n+1}^{(2)}=\sum_{k=1}^{\infty} \frac{1}{k^{2 n+1}} \sum_{j=1}^{2 k} \frac{1}{j} .
$$

Note that

$$
\begin{aligned}
E_{1,2 n+1}^{(2)}-S_{1,2 n+1} & =\sum_{k=1}^{\infty} \frac{1}{k^{2 n+1}} \sum_{j=k+1}^{2 k} \frac{1}{j} \\
& =\frac{1}{2} \zeta(2 n+2)+\sum_{k=1}^{\infty} \frac{1}{k^{2 n+1}} \sum_{j=1}^{k-1} \frac{1}{j+k} \\
& =\frac{1}{2} \zeta(2 n+2)+\sum_{k=1}^{\infty} \sum_{j=1}^{\infty} \frac{1}{(k+j)^{2 n+1}(k+2 j)} .
\end{aligned}
$$


In light of the partial fraction decomposition

$$
\begin{aligned}
\frac{1}{(x+k)^{2 n+1}(2 x+k)}= & \frac{2^{2 n}}{k^{2 n+1}}\left\{\frac{1}{x+k / 2}-\frac{1}{x+k}\right\}-\frac{1}{k(x+k)^{2 n+1}} \\
& -\sum_{\ell=2}^{2 n} \frac{2^{\ell-1}}{k^{\ell}(x+k)^{2 n+2-\ell}},
\end{aligned}
$$

we conclude that

$$
\begin{aligned}
\sum_{k=1}^{\infty} & \sum_{j=1}^{\infty} \frac{1}{(k+j)^{2 n+1}(k+2 j)} \\
= & 2^{2 n} \sum_{k=1}^{\infty} \frac{1}{k^{2 n+1}} \sum_{j=1}^{\infty}\left(\frac{1}{j+k / 2}-\frac{1}{j+k}\right)-\sum_{k=1}^{\infty} \sum_{j=1}^{\infty} \frac{1}{k(k+j)^{2 n+1}} \\
& -\sum_{\ell=2}^{2 n} 2^{\ell-1} \sum_{k=1}^{\infty} \sum_{j=1}^{\infty} \frac{1}{k^{\ell}(k+j)^{2 n+2-\ell}} .
\end{aligned}
$$

The second term in the above is equal to

$$
-\left\{S_{1,2 n+1}-\zeta(2 n+2)\right\},
$$

while the third term is equal to

$$
-\sum_{\ell=2}^{2 n} 2^{\ell-1}\left\{S_{\ell, 2 n+2-\ell}-\zeta(2 n+2)\right\},
$$

or

$$
-\sum_{\ell=2}^{2 n} 2^{\ell-1} S_{\ell, 2 n+2-\ell}+\left(2^{2 n}-2\right) \zeta(2 n+2) .
$$

Now it remains to evaluate the first term. When $k$ ranges over all positive even integers, the corresponding partial sum is equal to

$$
2^{2 n} \sum_{k=1}^{\infty} \frac{1}{(2 k)^{2 n+1}} \sum_{j=1}^{\infty}\left\{\frac{1}{j+k}-\frac{1}{j+2 k}\right\},
$$

which is equal to

$$
\frac{1}{2}\left\{E_{1,2 n+1}^{(2)}-S_{1,2 n+1}\right\} .
$$

When $k$ ranges over all positive odd integers, the corresponding partial sum is

$$
2^{2 n} \sum_{k=0}^{\infty} \frac{1}{(2 k+1)^{2 n+1}} \sum_{j=1}^{\infty}\left\{\frac{1}{j+k+1 / 2}-\frac{1}{j+2 k+1}\right\} .
$$


In view of the Kronecker limit formula for Hurwitz zeta function [1],

$$
\lim _{s \rightarrow 1^{+}}\left\{\zeta(s, \delta)-\frac{1}{s-1}\right\}=-\frac{\Gamma^{\prime}(\delta)}{\Gamma(\delta)},
$$

we get in particular that

$$
\sum_{j=1}^{\infty}\left\{\frac{1}{j+k+1 / 2}-\frac{1}{j+2 k+1}\right\}=-\frac{\Gamma^{\prime}(k+3 / 2)}{\Gamma(k+3 / 2)}+\frac{\Gamma^{\prime}(2 k+2)}{\Gamma(2 k+2)}
$$

Also we have for positive integers $k$ (e.g. see [1] ),

$$
-\frac{\Gamma^{\prime}(k+3 / 2)}{\Gamma(k+3 / 2)}=-2\left(1+\frac{1}{3}+\cdots+\frac{1}{2 k+1}\right)+\gamma+2 \log 2
$$

and

$$
\frac{\Gamma^{\prime}(2 k+2)}{\Gamma(2 k+2)}=1+\frac{1}{2}+\cdots+\frac{1}{2 k+1}-\gamma
$$

where $\gamma$ is the Euler constant defined by

$$
\gamma=\lim _{n \rightarrow \infty}\left(\sum_{k=1}^{n} \frac{1}{k}-\log n\right)
$$

Consequently, this expression in (2.4) is equal to

$$
2^{2 n+1} \lambda(2 n+1) \log 2-2^{2 n} \sum_{k=0}^{\infty} \frac{1}{(2 k+1)^{2 n+1}} \sum_{j=1}^{2 k+1} \frac{(-1)^{j+1}}{j},
$$

which we rewrite as

$$
2^{2 n+1} \lambda(2 n+1) \log 2-2^{2 n} H_{1,2 n+1}+2^{2 n-1} G_{1,2 n+1} .
$$

It follows that

$$
\begin{aligned}
\frac{1}{2} E_{1,2 n+1}^{(2)}= & -\frac{1}{2} S_{1,2 n+1}+\left(2^{2 n}-\frac{1}{2}\right) \zeta(2 n+2)+2^{2 n+1} \lambda(2 n+1) \log 2 \\
& -2^{2 n} H_{1,2 n+1}+2^{2 n-1} G_{1,2 n+1}-\sum_{\ell=2}^{2 n} 2^{\ell-1} S_{\ell, 2 n+2-\ell} .
\end{aligned}
$$

Our assertion then follows from the further relation

$$
\frac{1}{2}\left\{E_{1,2 n+1}^{(2)}+2^{2 n+1} H_{1,2 n+1}\right\}=2^{2 n} S_{1,2 n+1}-2^{2 n-1} G_{1,2 n+1} .
$$

As an immediate consequence, we have 
Proposition 2. For each positive integer n,

$$
\begin{aligned}
E_{1,2 n+1}^{(2)}+2^{2 n+1} H_{1,2 n+1}= & \left(2^{2 n}-\frac{1}{2}\right) S_{1,2 n+1}+\left(2^{2 n}-\frac{1}{2}\right) \zeta(2 n+2) \\
& +2^{2 n+1} \lambda(2 n+1) \log 2-\sum_{\ell=2}^{2 n} 2^{\ell-1} S_{\ell, 2 n+2-\ell} .
\end{aligned}
$$

Rremark. Unfortunately, both evaluations of $G_{1,2 n+1}$ and $E_{1,2 n+1}^{(2)}+2^{2 n+1} H_{1,2 n+1}$ involve the common expression

$$
\sum_{\ell=2}^{2 n} 2^{\ell-1} S_{\ell, 2 n+2-\ell}
$$

Such a sum can be replaced by a linear combination of $S_{1,2 n+1}$ and $\zeta(p) \zeta(q)$ with $p+q=$ $2 n+2$ as we shall see in Section 4 .

\section{Explicit evaluation of $E_{1,2 n+1}^{(2)}+2^{2 n+1} H_{1,2 n+1}$}

Our first attempt to evaluate $G_{1,2 n+1}$ is unsuccessful, so we try another way to deal with $E_{1,2 n+1}$ and $H_{1,2 n+1}$ directly.

Proposition 3. For each positive integer $n$,

$$
2^{-2 n}\left\{E_{1,2 n+1}^{(2)}-\frac{1}{2} S_{1,2 n+1}\right\}=\lambda(2 n+1) \log 2+\frac{1}{2} G_{1,2 n+1}-\sum_{j=2}^{2 n} 2^{-j} F_{2 n+2-j, j},
$$

where for $p, q \geq 2$,

$$
F_{p, q}=\sum_{k=1}^{\infty} \frac{1}{k^{q}} \sum_{j=0}^{k-1} \frac{1}{(2 j+1)^{p}}=E_{p, q}^{(2)}-2^{-p} S_{p, q}
$$

Proof. We begin with the difference

$$
E_{1,2 n+1}^{(2)}-\frac{1}{2} S_{1,2 n+1}=\sum_{k=1}^{\infty} \frac{1}{k^{2 n+1}} \sum_{j=0}^{k-1} \frac{1}{2 j+1}
$$

It follows that

$$
\begin{aligned}
2^{-(2 n+1)}\left\{E_{1,2 n+1}^{(2)}-\frac{1}{2} S_{1,2 n+1}\right\} & =\sum_{k=1}^{\infty} \frac{1}{(2 k)^{2 n+1}} \sum_{j=0}^{k-1} \frac{1}{2 j+1} \\
& =\sum_{k=0}^{\infty} \sum_{j=0}^{\infty} \frac{1}{(2 k+2 j+2)^{2 n+1}(2 j+1)}
\end{aligned}
$$


with a simple change of variable $k=k^{\prime}+j+1$ in the summation. We need the partial fraction decomposition

$$
\begin{aligned}
\frac{1}{(x+2 k+1)^{2 n+1} x}= & \frac{1}{(2 k+1)^{2 n+1}}\left\{\frac{1}{x}-\frac{1}{x+2 k+1}\right\}-\frac{1}{(2 k+1)(x+2 k+1)^{2 n+1}} \\
& -\sum_{\ell=2}^{2 n} \frac{1}{(2 k+1)^{\ell}(x+2 k+1)^{2 n+2-\ell}} .
\end{aligned}
$$

Setting $x=2 j+1$ and letting $j$ and $k$ range over all non-negative integers, we get

$$
\begin{aligned}
2^{-(2 n+1)}\left\{E_{1,2 n+1}^{(2)}-\frac{1}{2} S_{1,2 n+1}\right\}= & \sum_{k=0}^{\infty} \frac{1}{(2 k+1)^{2 n+1}} \sum_{j=0}^{\infty}\left\{\frac{1}{2 j+1}-\frac{1}{2 j+2 k+2}\right\} \\
& -\sum_{k=0}^{\infty} \sum_{j=0}^{\infty} \frac{1}{(2 k+1)(2 j+2 k+2)^{2 n+1}} \\
& -\sum_{\ell=2}^{2 n} \sum_{k=0}^{\infty} \sum_{j=0}^{\infty} \frac{1}{(2 k+1)^{\ell}(2 k+2 n+2)^{2 n+2-\ell}} .
\end{aligned}
$$

The second term on the right-hand side is equal to

$$
-2^{-(2 n+1)}\left\{E_{1,2 n+1}^{(2)}-\frac{1}{2} S_{1,2 n+1}\right\},
$$

while the third term is equal to

$$
-\sum_{\ell=2}^{2 n} 2^{-\ell} F_{2 n+2-\ell, \ell} .
$$

The inner summation of the first term is equal to

$$
\frac{1}{2}\left\{-\frac{\Gamma^{\prime}(1 / 2)}{\Gamma(1 / 2)}+\frac{\Gamma^{\prime}(k+1)}{\Gamma(k+1)}\right\},
$$

hence the whole term is equal to

$$
\lambda(2 n+1) \log 2+\frac{1}{2} G_{1,2 n+1} .
$$

Thus our assertion follows.

In a similar way, we get

Proposition 4. For each positive integer $n$,

$$
\begin{aligned}
H_{1,2 n+1}= & \lambda(2 n+2)+2^{-(2 n+1)}\left\{E_{1,2 n+1}^{(2)}-\frac{1}{2} S_{1,2 n+1}\right\}-\frac{1}{2} G_{1,2 n+1} \\
& -\sum_{\ell=2}^{2 n} 2^{-\ell} G_{l, 2 n+2-\ell .}
\end{aligned}
$$


Proof. We begin with the definition of $H_{1,2 n+1}$ :

$$
H_{1,2 n+1}=\sum_{k=0}^{\infty} \frac{1}{(2 k+1)^{2 n+1}} \sum_{j=0}^{k} \frac{1}{2 j+1}
$$

which we rewrite as

$$
\sum_{k=0}^{\infty} \sum_{j=0}^{\infty} \frac{1}{(2 k+2 j+1)^{2 n+1}(2 j+1)}=\lambda(2 n+2)+\sum_{k=1}^{\infty} \sum_{j=0}^{\infty} \frac{1}{(2 k+2 j+1)^{2 n+1}(2 j+1)}
$$

In order to evaluate the double series, we need the partial fraction decomposition:

$$
\begin{aligned}
\frac{1}{(2 k+x)^{2 n+1} x}= & \frac{1}{(2 k)^{2 n+1}}\left\{\frac{1}{x}-\frac{1}{x+2 k}\right\}-\frac{1}{2 k(2 k+x)^{2 n}} \\
& -\sum_{\ell=2}^{2 n} \frac{1}{(2 k)^{\ell}(2 k+x)^{2 n+2-\ell}} .
\end{aligned}
$$

Setting $x=2 j+1$ and letting $j$ range over all non-negative integers and $k$ range over all positive integers, we get

$$
\begin{aligned}
H_{1,2 n+1}= & \lambda(2 n+2)+\sum_{k=1}^{\infty} \frac{1}{(2 k)^{2 n+1}} \sum_{j=0}^{\infty}\left\{\frac{1}{2 j+1}-\frac{1}{2 j+2 k+1}\right\} \\
& -\sum_{k=1}^{\infty} \sum_{j=0}^{\infty} \frac{1}{2 k(2 k+2 j+1)^{2 n}}-\sum_{\ell=2}^{2 n} \frac{1}{(2 k)^{\ell}(2 k+2 j+1)^{2 n+2-\ell}}
\end{aligned}
$$

The second term in the right-hand side can be rewritten as

$$
2^{-(2 n+1)} \sum_{k=1}^{\infty} \frac{1}{k^{2 n+1}} \sum_{j=0}^{k-1} \frac{1}{2 j+1}
$$

which is equal to

$$
2^{-(2 n+1)}\left\{E_{1,2 n+1}^{(2)}-\frac{1}{2} S_{1,2 n+1}\right\} .
$$

On the other hand, simply by the definition of $G_{p, q}$, the third term and final term are equal to $-\frac{1}{2} G_{1,2 n+1}$ and $-\sum_{\ell=2}^{2 n} 2^{-\ell} G_{\ell, 2 n+2-\ell}$, respectively. This proves the assertion.

Proof of Theorems 1, 2, and 3. Observe that for $p \geq 2$ and $q \geq 2$,

$$
2^{-p} G_{p, q}+2^{-p} F_{q, p}=\sum_{k=0}^{\infty} \frac{1}{(2 k+1)^{q}} \sum_{j=1}^{\infty} \frac{1}{(2 j)^{p}}=2^{-p} \zeta(p) \lambda(q) .
$$


So if we add formulas in Propositions 3 and 4 together, we get

$$
\begin{aligned}
2^{-(2 n+1)} E_{1,2 n+1}^{(2)}+H_{1,2 n+1}= & \lambda(2 n+1) \log 2+\lambda(2 n+2)+2^{-(2 n+2)} S_{1,2 n+1} \\
& -\sum_{\ell=2}^{2 n} 2^{-\ell} \zeta(\ell) \lambda(2 n+2-\ell) .
\end{aligned}
$$

Upon multiplying by $2^{2 n+1}$ on both sides, we prove Theorem 2 . By comparing two different expressions for $E_{1,2 n+1}^{(2)}+2^{2 n+1} H_{1,2 n+1}$ in Proposition 2 and Theorem 2 , we get

$$
\begin{aligned}
\sum_{\ell=2}^{2 n} 2^{\ell-1} S_{\ell, 2 n+2-\ell}= & \left(2^{2 n}-1\right) S_{1,2 n}-2^{2 n} \zeta(2 n+2) \\
& +\sum_{\ell=2}^{2 n}\left(2^{2 n+1-\ell}-\frac{1}{2}\right) \zeta(\ell) \zeta(2 n+2-\ell),
\end{aligned}
$$

which proves Theorem 3. Combining Theorem 3 and Proposition 1 then proves Theorem 1.

\section{Relations among Euler sums of the same even weight}

Some linear relations among Euler sums $S_{\ell, 2 n+2-\ell}, \ell=2,3, \ldots, 2 n$ can be obtained easily from the partial fraction decompositions of the rational functions

$$
\frac{1}{x^{\ell}(x+1)^{2 n+2-\ell}}, \quad \ell=2,3, \ldots, 2 n .
$$

Here we illustrate the details. It is easy to see that

$$
\frac{1}{x^{2}(x+1)^{2 n}}=\frac{1}{x^{2}}-\frac{2 n}{x}+\frac{2 n}{x+1}+\frac{2 n-1}{(x+1)^{2}}+\cdots+\frac{1}{(x+1)^{2 n}} .
$$

Replacing $x$ by $x / k$, we get another identity ready to be used

$$
\frac{1}{x^{2}(x+k)^{2 n}}=\frac{1}{k^{2 n} x^{2}}-\frac{2 n}{k^{2 n+1}}\left\{\frac{1}{x}-\frac{1}{x+k}\right\}+\sum_{\ell=2}^{2 n} \frac{\ell-1}{k^{\ell}(x+k)^{2 n+2-\ell}} .
$$

As $x$ and $k$ both range over all positive integers and sum together, we get

$$
S_{2,2 n}-\zeta(2 n+2)=\zeta(2) \zeta(2 n)-2 n S_{1,2 n+1}+\sum_{\ell=2}^{2 n}(\ell-1)\left\{S_{\ell, 2 n+2-\ell}-\zeta(2 n+2)\right\}
$$

which we rewrite as

$$
S_{2,2 n}+2 S_{3,2 n-1}+3 S_{4,2 n-2}+\cdots+(2 n-1) S_{2 n, 2}=S_{2,2 n}+\Delta(2),
$$


where $\Delta(2)$ is a linear combination of $\zeta(2 n+2), \zeta(2) \zeta(2 n)$ and $S_{1,2 n+1}$.

On the other hand, from the partial fraction decomposition

$$
\begin{aligned}
\frac{1}{x^{3}(x+1)^{2 n-1}}= & \frac{1}{x^{3}}-\frac{2 n-1}{x^{2}}+\frac{2 n(2 n-1)}{2}\left\{\frac{1}{x}-\frac{1}{x+1}\right\} \\
& -\sum_{\ell=2}^{2 n-2} \frac{\ell(\ell-1)}{2} \frac{1}{(x+1)^{\ell}},
\end{aligned}
$$

we get the identity

$$
\begin{aligned}
\frac{1}{x^{3}(x+k)^{2 n-1}}= & \frac{1}{k^{2 n-1} x^{3}}-\frac{2 n-1}{k^{2 n} x^{2}}+\frac{2 n(2 n-1)}{2} \frac{1}{k^{2 n+1}}\left\{\frac{1}{x}-\frac{1}{x+k}\right\} \\
& -\sum_{\ell=2}^{2 n-1} \frac{(2 n-\ell)(2 n-\ell-1)}{2} \frac{1}{k^{2 n+2-\ell}(x+k)^{\ell}} .
\end{aligned}
$$

Consequently, we have another relation

$$
S_{3,2 n-1}+3 S_{4,2 n-2}+6 S_{5,2 n-3}+\cdots+\frac{(2 n-2)(2 n-3)}{2} S_{2 n, 2}=-S_{3,2 n-1}+\Delta(3),
$$

where $\Delta(3)$ is a linear combination of $\zeta(2 n+2), \zeta(2) \zeta(2 n), \zeta(3) \zeta(2 n-1)$ and $S_{1,2 n+1}$.

Continuing this process, we get the following system of linear relations among $S_{\ell, 2 n+2-\ell}, \ell=2,3, \ldots, 2 n$

$$
\left\{\begin{aligned}
S_{2,2 n}+2 S_{3,2 n-1}+3 S_{4,2 n-2}+4 S_{5,2 n-3}+\cdots+(2 n-1) S_{2 n, 2} & =S_{2,2 n}+\Delta(2) \\
S_{3,2 n-1}+3 S_{4,2 n-2}+6 S_{5,2 n-3}+\cdots+\left(\begin{array}{c}
2 n-1 \\
2
\end{array}\right) S_{2 n, 2} & =-S_{3,2 n-1}+\Delta(3) \\
S_{4,2 n-2}+4 S_{5,2 n-3}+\cdots+\left(\begin{array}{c}
2 n-1 \\
3
\end{array}\right) S_{2 n, 2} & =S_{4,2 n-2}+\Delta(4) \\
\cdots \cdots \cdots \cdots & \\
S_{2 n-1,3}+(2 n-1) S_{2 n, 2} & =-S_{2 n-1,3}+\Delta(2 n-1) \\
S_{2 n, 2} & =S_{2 n, 2}+\Delta(2 n) .
\end{aligned}\right.
$$

All adding together, we obtain

$$
\sum_{\ell=2}^{2 n}\left(2^{\ell-1}-1\right) S_{\ell, 2 n+2-\ell}=\sum_{\ell=2}^{2 n}(-1)^{\ell} S_{\ell, 2 n+2-\ell}+\sum_{\ell=2}^{2 n} \Delta(\ell) .
$$

Therefore,

$$
\sum_{\ell=2}^{2 n} 2^{\ell-1} S_{\ell, 2 n+2-\ell}=\sum_{\ell=2}^{2 n} S_{\ell, 2 n+2-\ell}+\sum_{\ell=2}^{2 n}(-1)^{\ell} S_{\ell, 2 n+2-\ell}+\sum_{\ell=2}^{2 n} \Delta(\ell) .
$$


Note that

$$
\sum_{\ell=2}^{2 n} S_{\ell, 2 n+2-\ell}=\frac{1}{2} \sum_{\ell=2}^{2 n} \zeta(\ell) \zeta(2 n+2-\ell)-(2 n-1) \zeta(2 n)
$$

and

$$
\sum_{\ell=2}^{2 n}(-1)^{\ell} S_{\ell, 2 n+2-\ell}=\frac{1}{2} \sum_{\ell=2}^{2 n}(-1)^{\ell} \zeta(\ell) \zeta(2 n+2-\ell)+ \begin{cases}\frac{1}{2} \zeta(2 n+2), & \text { if } n \text { is odd } \\ -\frac{1}{2} \zeta(2 n+2), & \text { if } n \text { is even }\end{cases}
$$

So it is not a surprise that the sum

$$
\sum_{\ell=2}^{2 n} 2^{\ell-1} S_{\ell, 2 n+2-\ell}
$$

can be expressed in terms of $\zeta(p) \zeta(q)$ with $p+q=2 n+2$ and $S_{1,2 n+1}$.

Remark. If we treat $S_{2,2 n}, S_{3,2 n-1}, \ldots, S_{2 n, 2}$ as $2 n-1$ unknowns, the coefficient matrix of the system is given by

$$
\left[\begin{array}{ccccccc}
0 & 2 & 3 & 4 & \ldots & \ldots & 2 n-1 \\
0 & 2 & 3 & 6 & \ldots & \ldots & \left(\begin{array}{c}
2 n-1 \\
2
\end{array}\right) \\
0 & 0 & 0 & 4 & \ldots & \ldots & \left(\begin{array}{c}
2 n-1 \\
3
\end{array}\right) \\
\ldots & \ldots & \ldots & \ldots & \ldots & \ldots & \ldots \ldots \\
\ldots & \ldots & \ldots & \ldots & \ldots & 2 & 2 n-1 \\
0 & 0 & 0 & 0 & 0 & 0 & 0
\end{array}\right]
$$

The rank of this matrix is $n-2$. But still we have the relation

$$
S_{p, q}+S_{q, p}=\zeta(p) \zeta(q)+\zeta(p+q),
$$

and so

$$
S_{n+1, n+1}=\frac{1}{2}\left\{\zeta^{2}(n+1)+\zeta(2 n+2)\right\} .
$$

So there are actually $n-1$ unknowns $S_{2,2 n}, S_{3,2 n-1}, \ldots, S_{n, n+2}$ to be determined from only $n-2$ independent linear equations. Of course, the result is undetermined. However, we are able to evaluate suitable linear combinations of a pair of sums. For example, when $n=5$, we have 4 unknowns $S_{2,10}, S_{3,9}, S_{4,8}, S_{5,7}$ subject to three conditions

$$
\left\{\begin{aligned}
9 S_{2,10}+6 S_{3,9}+4 S_{4,8}+2 S_{5,7} & =\Delta(2) \\
36 S_{2,10}+26 S_{3,9}+18 S_{4,8}+9 S_{5,7} & =\Delta(3) \\
84 S_{2,10}+56 S_{3,9}+35 S_{4,8}+16 S_{5,7} & =\Delta(4) .
\end{aligned}\right.
$$

The above system is equivalent to

$$
\left\{\begin{aligned}
9 S_{2,10}+2 S_{3,9} & =\Delta^{\prime}(2) \\
16 S_{3,9}+9 S_{4,8} & =\Delta^{\prime}(3) \\
7 S_{4,8}+8 S_{5,7} & =\Delta^{\prime}(4) .
\end{aligned}\right.
$$


Consequently, we are able to express $9 S_{2,10}+2 S_{3,9}, 16 S_{3,9}+9 S_{4,8}$ and $7 S_{4,8}+8 S_{5,7}$ explicitly in terms of values of the Riemann zeta function at positive integers.

Exactly the same happens to $E_{1,3}^{(2)}, H_{1,3}$ and $G_{2,2}$. By Theorem 2, we have

or

$$
E_{1,3}^{(2)}+8 H_{1,3}=\frac{1}{2} S_{1,3}+\frac{15}{2} \zeta(4)+8 \lambda(3) \log 2-2 \zeta(2) \lambda(2)
$$

$$
E_{1,3}^{(2)}+8 H_{1,3}=\frac{35}{8} \zeta(4)+7 \zeta(3) \log 2 .
$$

Also from Proposition 4, we get

$$
E_{1,3}^{(2)}-8 H_{1,3}-2 G_{2,2}=-\frac{5}{4} \zeta(4)-7 \zeta(3) \log 2 .
$$

With only (4.14) and (4.15) at our disposal, we are unable to determine $E_{1,3}^{(2)}, H_{1,3}$ and $G_{2,2}$ individually.

The above discussion indicates the indeterminacy nature of Ramanujan's constant $G(1)$, which is due to the lack of linearly independent relations among Euler sums of the same even weight.

\section{A further generalization}

For a pair of positive integers $p$ and $q$ with $q \geq 2$ and another pair of positive integers $a$ and $b$, we define new sums

$$
G_{p, q}(a, b)=\sum_{k=0}^{\infty} \frac{1}{(a k+b)^{q}} \sum_{j=1}^{k} \frac{1}{j^{p}}
$$

and

$$
H_{p, q}(a, b)=\sum_{k=0}^{\infty} \frac{1}{(a k+b)^{q}} \sum_{j=0}^{k} \frac{1}{(a j+b)^{p}} .
$$

When $a=2, b=1$ and $p+q$ is odd, the evaluations of $G_{p, q}(2,1)$ and $H_{p, q}(2,1)$ are already known [6]. Here we shall give the evaluations of $G_{1, n}(a, b)$ and

$$
E_{1, n}^{(a)}+a^{n} \sum_{b=1}^{a-1} H_{1, n}(a, b)
$$

through relations among $G_{p, q}(a, b), H_{p, q}(a, b)$ and $E_{p, q}^{(a)}$.

Theorem 4. For positive integers $n \geq 2$ and a pair of positive integers a and $b$, we have

$$
\begin{aligned}
G_{1, n}(a, b)= & \frac{n}{2 a^{n}} \zeta\left(n+1, \frac{b}{a}\right)+\frac{1}{a^{n}} \zeta\left(n, \frac{b}{a}\right)\left\{\frac{\Gamma^{\prime}(b / a)}{\Gamma(b / a)}+\gamma\right\} \\
& -\frac{1}{2 a^{n}} \sum_{\ell=2}^{n-1} \zeta\left(\ell, \frac{b}{a}\right) \zeta\left(n+1-\ell, \frac{b}{a}\right) .
\end{aligned}
$$


Proof. Right from the definition (5.1), we have

$$
a^{-1} G_{1, n}(a, b)=\sum_{k=0}^{\infty} \frac{1}{(a k+b)^{n}} \sum_{j=1}^{k} \frac{1}{a j}=\sum_{k=0}^{\infty} \sum_{j=1}^{\infty} \frac{1}{(a k+a j+b)^{n} a j} .
$$

We need the following partial fraction decomposition

$$
\frac{1}{(x+\alpha)^{n} x}=\frac{1}{\alpha^{n}}\left\{\frac{1}{x}-\frac{1}{x+\alpha}\right\}-\frac{1}{\alpha(x+\alpha)^{n}}-\sum_{\ell=2}^{n-1} \frac{1}{\alpha^{\ell}(x+\alpha)^{n+1-\ell}} .
$$

Set $x=a j$ and $\alpha=a k+b$. Then letting $j$ range over all positive integers, $k$ range over all non-negative integers and summing together, we get

$$
\begin{aligned}
a^{-1} G_{1, n}(a, b)= & \sum_{k=0}^{\infty} \frac{1}{(a k+b)^{n}} \sum_{j=1}^{\infty}\left\{\frac{1}{a j}-\frac{1}{a k+a j+b}\right\} \\
& -\sum_{k=0}^{\infty} \sum_{j=1}^{\infty} \frac{1}{(a k+b)(a k+a j+b)^{n}} \\
& -\sum_{\ell=2}^{n-1} \sum_{k=0}^{\infty} \sum_{j=1}^{\infty} \frac{1}{(a k+b)^{\ell}(a k+a j+b)^{n+1-\ell}} .
\end{aligned}
$$

The first term is equal to

$$
\frac{1}{a} \sum_{k=0}^{\infty} \frac{1}{(a k+b)^{n}}\left\{\frac{\Gamma^{\prime}(k+1+b / a)}{\Gamma(k+1+b / a)}-\frac{\Gamma^{\prime}(1)}{\Gamma(1)}\right\}
$$

which we rewrite as

$$
\sum_{k=0}^{\infty} \frac{1}{(a k+b)^{n}} \sum_{j=0}^{k} \frac{1}{a j+b}+\frac{1}{a} \sum_{k=0}^{\infty} \frac{1}{(a k+b)^{n}}\left\{\frac{\Gamma^{\prime}(b / a)}{\Gamma(b / a)}+\gamma\right\}
$$

or simply as

$$
H_{1, n}(a, b)+\frac{1}{a^{n+1}} \zeta\left(n, \frac{b}{a}\right)\left\{\frac{\Gamma^{\prime}(b / a)}{\Gamma(b / a)}+\gamma\right\} .
$$

The second term is equal to the negative of

$$
H_{1, n}(a, b)-\frac{1}{a^{n+1}} \zeta\left(n+1, \frac{b}{a}\right)
$$

and the third term is equal to the negative of

$$
\sum_{\ell=2}^{n-1}\left\{H_{\ell, n+1-\ell}(a, b)-\frac{1}{a^{n+1}} \zeta\left(n+1, \frac{b}{a}\right)\right\} .
$$


Consequently, we have

$$
\begin{aligned}
G_{1, n}(a, b)= & \frac{n-1}{a^{n}} \zeta\left(n+1, \frac{b}{a}\right)+\frac{1}{a^{n}} \zeta\left(n, \frac{b}{a}\right)\left\{\frac{\Gamma^{\prime}(b / a)}{\Gamma(b / a)}+\gamma\right\} \\
& -a \sum_{\ell=2}^{n-1} H_{\ell, n+1-\ell}(a, b) .
\end{aligned}
$$

Our assertion then follows from

$$
\begin{aligned}
\sum_{\ell=2}^{n-1} H_{\ell, n+1-\ell}(a, b) & =\frac{1}{2} \sum_{\ell=2}^{n-1}\left\{H_{\ell, n+1-\ell}(a, b)+H_{n+1-\ell, \ell}(a, b)\right\} \\
& =\frac{1}{2 a^{n+1}} \sum_{\ell=2}^{n-1}\left\{\zeta\left(\ell, \frac{b}{a}\right) \zeta\left(n+1-\ell, \frac{b}{a}\right)+\zeta\left(n+1, \frac{b}{a}\right)\right\}
\end{aligned}
$$

Theorem 5. For positive integers $n$ and $a$ with $n \geq 2, a \geq 2$, we have

$$
\begin{aligned}
E_{1, n}^{(a)}+a^{n} \sum_{b=1}^{a-1} H_{1, n}(a, b)= & a^{-1} S_{1, n}+\left(a^{n}-\frac{1}{a}\right) \zeta(n+1)-\sum_{b=1}^{a-1} \zeta\left(n, 1-\frac{b}{a}\right)\left\{\frac{\Gamma^{\prime}(b / a)}{\Gamma(b / a)}+\gamma\right\} \\
& -\sum_{b=1}^{a-1} \sum_{\ell=2}^{n-1} a^{-1} \zeta(\ell) \zeta\left(n+1-\ell, \frac{b}{a}\right)
\end{aligned}
$$

Proof. At first, we have

$$
\begin{aligned}
H_{1, n}(a, b) & =\sum_{k=0}^{\infty} \sum_{j=0}^{\infty} \frac{1}{(a k+a j+b)^{n}(a j+b)} \\
& =a^{-(n+1)} \zeta\left(n+1, \frac{b}{a}\right)+\sum_{k=1}^{\infty} \sum_{j=0}^{\infty} \frac{1}{(a k+a j+b)^{n}(a j+b)}
\end{aligned}
$$

With the help of partial fraction decomposition, the second term is equal to

$$
\sum_{k=1}^{\infty} \frac{1}{(a k)^{n}} \sum_{j=0}^{\infty}\left\{\frac{1}{a j+b}-\frac{1}{a k+a j+b}\right\}-\sum_{\ell=1}^{n-1} \frac{1}{(a k)^{\ell}(a k+a j+b)^{n+1-\ell}}
$$

or

$$
a^{-n} \sum_{k=1}^{\infty} \frac{1}{k^{n}} \sum_{j=0}^{k-1} \frac{1}{a j+b}-\sum_{\ell=1}^{n-1} a^{-\ell} G_{\ell, n+1-\ell}(a, b) .
$$


Let $b$ range over $1,2, \ldots, a-1$ and sum together, we get

$$
\begin{aligned}
\sum_{b=1}^{a-1} H_{1, n}(a, b)= & \sum_{b=1}^{a-1} a^{-(n+1)} \zeta\left(n+1, \frac{b}{a}\right)+a^{-n}\left\{E_{1, n}^{(a)}-\frac{1}{a} S_{1, n}\right\} \\
& -\sum_{b=1}^{a-1} \sum_{\ell=1}^{n-1} a^{-\ell} G_{\ell, n+1-\ell}(a, b) .
\end{aligned}
$$

On the other hand, we have

$$
\begin{aligned}
a^{-n}\left\{E_{1, n}^{(a)}-a^{-1} S_{1, n}\right\} & =\sum_{k=1}^{\infty} \frac{1}{(a k)^{n}}\left\{\sum_{j=1}^{a k} \frac{1}{j}-\sum_{j=1}^{k} \frac{1}{a j}\right\} \\
& =\sum_{b=1}^{a-1} \sum_{k=1}^{\infty} \frac{1}{(a k)^{n}} \sum_{j=0}^{k-1} \frac{1}{a j+b} \\
& =\sum_{b=1}^{a-1} \sum_{k=0}^{\infty} \sum_{j=0}^{\infty} \frac{1}{(a k+a j+a)^{n}(a j+b)}
\end{aligned}
$$

As before, we employ partial fraction decomposition to get

$$
\begin{aligned}
\sum_{k=0}^{\infty} \sum_{j=0}^{\infty} \frac{1}{(a k+a j+a)^{n}(a j+b)}= & a^{-1} G_{1,2 n}(a, a-b)-a^{-n} \zeta\left(n, 1-\frac{b}{a}\right)\left\{\frac{\Gamma^{\prime}(b / a)}{\Gamma(b / a)}+\gamma\right\} \\
& -\sum_{\ell=1}^{n-1} \sum_{k=1}^{\infty} \frac{1}{(a k)^{n+1-\ell}} \sum_{j=0}^{k-1} \frac{1}{(a j+a-b)^{\ell}} .
\end{aligned}
$$

So it follows that

$$
\begin{aligned}
2 a^{-n}\left\{E_{1, n}^{(a)}-a^{-1} S_{1, n}\right\}= & a^{-1} \sum_{b=1}^{a-1} G_{1,2 n}(a, a-b)-a^{-n} \sum_{b=1}^{a-1} \zeta\left(n, 1-\frac{b}{a}\right)\left\{\frac{\Gamma^{\prime}(b / a)}{\Gamma(b / a)}+\gamma\right\} \\
& -\sum_{\ell=2}^{n-1} a^{-\ell}\left\{E_{n+1-\ell, \ell}^{(a)}-a^{-(n+1)+\ell} S_{n+1-\ell, \ell}\right\} .
\end{aligned}
$$

Our assertion then follows from

$$
\left\{E_{p, q}^{(a)}-a^{-p} S_{p, q}\right\}+\sum_{b=1}^{a-1} a^{-q} G_{q, p}(a, b)=\sum_{b=1}^{a-1} a^{-(p+q)} \zeta(p) \zeta\left(q, \frac{b}{a}\right) .
$$

\section{Acknowledgements}

The author thanks his colleague, Minking Eie, for the support and encouragement. The author is also grateful for the support from the National Science Council of Taiwan, Republic of China, through grants NSC94-2115-M-194-009 and NSC95-2115-M-194-012. 


\section{References}

[1] B. C. Berndt, Ramanujan's Notebooks, Part I, Springer-Verlag, New York, 1985.

[2] D. Borwein, J. M. Borwein and R. Girgensohn, Explicit evaluation of Euler sums, Proc. Edinburgh Math. Soc. (2) 38(1995), 277-294.

[3] R. E. Crandall and J. P. Buhler, On the evaluation of Euler sums, Experiment. Math. 3(1994), 275-285.

[4] K.-W. Chen and M. Eie, Explicit evaluations of extended Euler sums, J. Number Theory $\mathbf{1 1 7}(2006), 31-52$.

[5] R. Sitaramachandrarao, A formula of S. Ramanujan, J. Number Theory 25(1987), 1-19.

[6] F.-Y. Yang, Bernoulli identities and application to Euler sums, Ph.D. dissertation, National Chung Cheng University, Minhsiung, Chiayi, Taiwan, 2004.

Department of Mathematics, National Chung Cheng University, Minhsiung, Chiayi 62145, Taiwan

E-mail: wcliaw@math.ccu.edu.tw 